\title{
Desktop system for accounting, audit, and research in A\&E
}

\author{
C J Taylor, S G Brain, F Bull, A C Crosby, D G Ferguson
}

\begin{abstract}
The development of a database for audit, research, and accounting in accident and emergency (A\&E) is described. The system uses a desktop computer, an optical scanner, sophisticated optical mark reader software, and workload management data. The system is highly flexible, easy to use, and at a cost of around $£ 16000$ affordable for larger departments wishing to move towards accounting. For smaller departments, it may be an alternative to full computerisation.

(F Accid Emerg Med 1997;14:92-97)
\end{abstract}

Keywords: accident and emergency department; audit; computer; workload management.

Most larger computerised accident and emergency (A\&E) systems in this country, whether they are custom designed or form part of the patient administration system (PAS), have one problem in common: once installed, they are difficult to change.

Although it may be easy to add another computer terminal, updating an existing system to capture new data usually requires the help of a software firm, which is costly and time consuming. When the new data are only to be used for a short term project, the expense is rarely justified and for one-off studies, like many audit projects, it is often easier to collect the data by hand than to write a software module to do the task. Most notably, most existing $\mathrm{A} \& \mathrm{E}$ computer systems were installed before

Royal Hallamshire Hospital, CSUH NHS Trust, Glossop Road Sheffield S10 2JF, United Kingdom: Accident and Emergency Department C J Taylor A C Crosby

D G Ferguson

\section{Department of}

Medical Audit

S G Brain

Workload Management Coordinator F Bull

Correspondence to: C J Taylor, Consultant in A\&E, James Paget Hospital, Great Yarmouth, Norfolk NR31 6LA.

Accepted for publication 16 July 1996 the NHS became cost conscious and none of these, as far as we are aware, has software modules capable of producing accurate coatings of individual patient episodes.

Using the workload management data outlined in a previous study, ${ }^{1}$ we developed a system that solves these problems. It is suitable for all $A \& E$ departments, irrespective of size.

\section{The system}

The system uses a desktop computer, an optical scanner, and three commercially available programs which can share and exchange data. Data sources are administrative information downloaded from PAS, clinical information collected at the time of patient attendance, and the workload management data derived from the study described by Taylor et al. ${ }^{1}$ Figure 1 represents graphically the steps necessary in the development and creation of the computerised database.

\section{HARDWARE AND SOFTWARE REQUIREMENTS}

Hardware

Those unfamiliar with computer jargon may simply wish to note that we used a state of the art desktop computer with a little mouse and a big screen (fig 2A). The computer was connected to an optical scanner (fig 2B) which takes an image of the questionnaire and sends this to the Formic ${ }^{\circledR}$ Software (see below) for processing. Formic then decides if any of the boxes have been ticked and keeps the images of any handwritten information for the user to view on-screen and code later.

The system runs on a standalone IBM compatible PC (dan 486DX2/66, $16 \mathrm{Mb}$ RAM, $525 \mathrm{Mb}$ hard disk and built in tape streamer) and a $17^{\prime}$ XGA monitor with $1024 \times 768$ resolution. The optical scanner is a Fujitsu M3093E, which reads single sided questionnaires and with the use of an ADF (automatic document feeder) is capable of scanning up to 23 pages $/ \mathrm{min}$.

\section{Software}

The system requires a database, a spreadsheet, and optical mark reader software. For database and spreadsheet, we used the Windows ${ }^{\circledR}$ versions of Access ${ }^{\circledR}$ and Excel $^{\circledR}$ respectively. Both programs are commercially available and come with their own documentation. Although probably unintelligible for those unused to computerised data handling, both are so widely known that most hospitals will have an IT technician on site who will be able to set up the system and customise both programs within a few hours.

The heart of the unit is Formic, a sophisticated optical mark reader program that fulfils the triple function of designing the scanner readable forms, driving the scanner and processing the data.

Formic reliably reads forms filled in biro, ink, and pencil. It ignores all information

Approximate cost for a middle of the range computer system (including $V A T$ )

\section{Non-recurrent}

Computer

Tape streamer

17' XGA monitor

Database and spreadsheet software

Optical scanner

OMR software

Training and installation

Total

Recurrent

Support for OMR

$£ 15850$

Support for scanner

On-site maintenance for other hardware Total 
outside defined areas of the sheet: smudges, too boldly marked tick boxes, and even doodles do not interfere with the scanning process. The user only has to keep filling the ADF; all errors encountered during scanning are stored as images for later on-screen editing.

The table gives a breakdown of the costs of the whole system.

THE PROCESS

The system was designed for maximum flexibility, and the steps outlined below should allow each department to customise it to their own requirements, including collecting a different dataset, using different forms, and extracting different statistics.

From choosing the dataset to the scanner readable form

One of the problems of collecting data for $A \& E$ is that the spread of patient conditions varies widely: to design a form that captures (in tick box form) all the information that should be collected for a multiply injured patient but

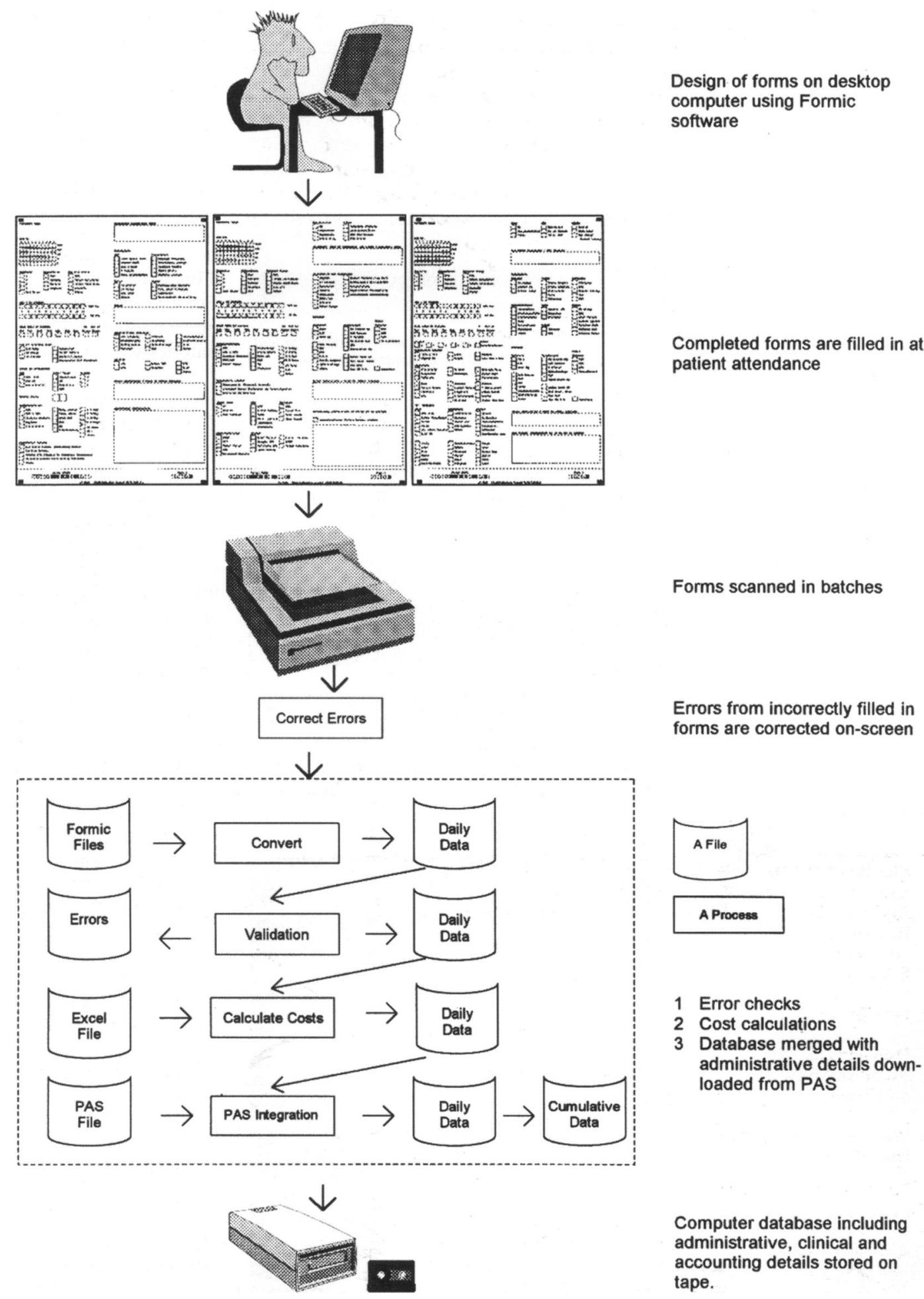

Figure 1 Flow chart representing the steps necessary in the development and creation of a computerised database for audit and accounting in $A \mathcal{E} E$. 

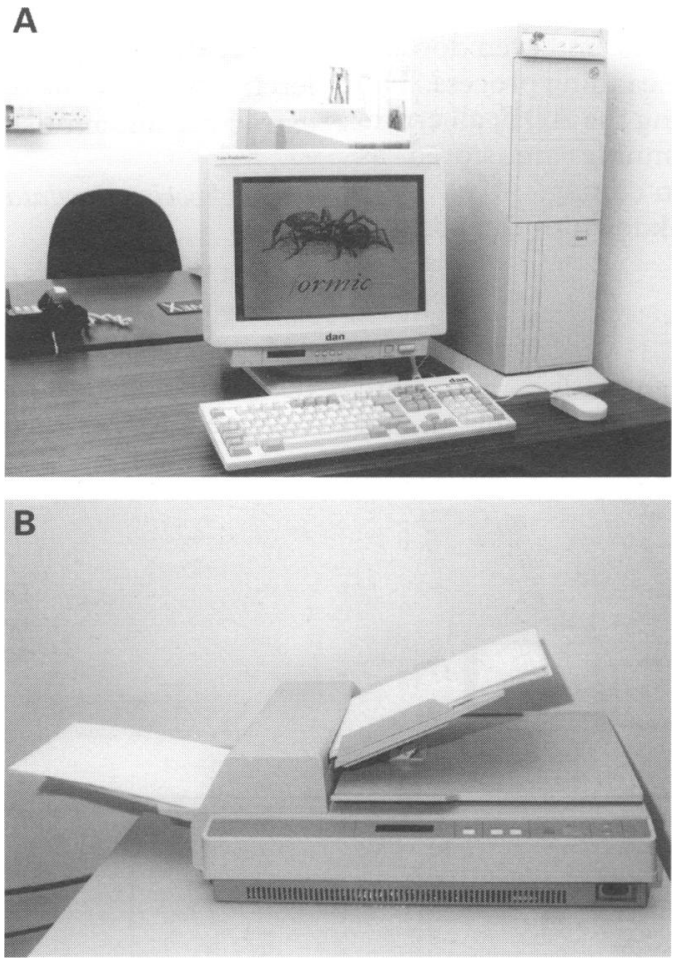

Figure 2 (A) The desktop computer used for development; (B) Optical scanner with ADF (automatic document feeder).

which is at the same time still simple enough to code the much more common complaint of "sprained ankle" is impossible. We solved the problem by designing three different forms, one for each of the traditional working areas of an A\&E department: resuscitation room, trolley bay, and walking wounded. As Formic does not require the completed forms to be sorted before scanning, this does not represent a practical problem.

Before determining the detail to be asked for on each sheet, we established the most common conditions, treatments, and outcomes in each of the three areas. With that, we were able to design forms that allow between $85 \%$ and $95 \%$ of all necessary information to be

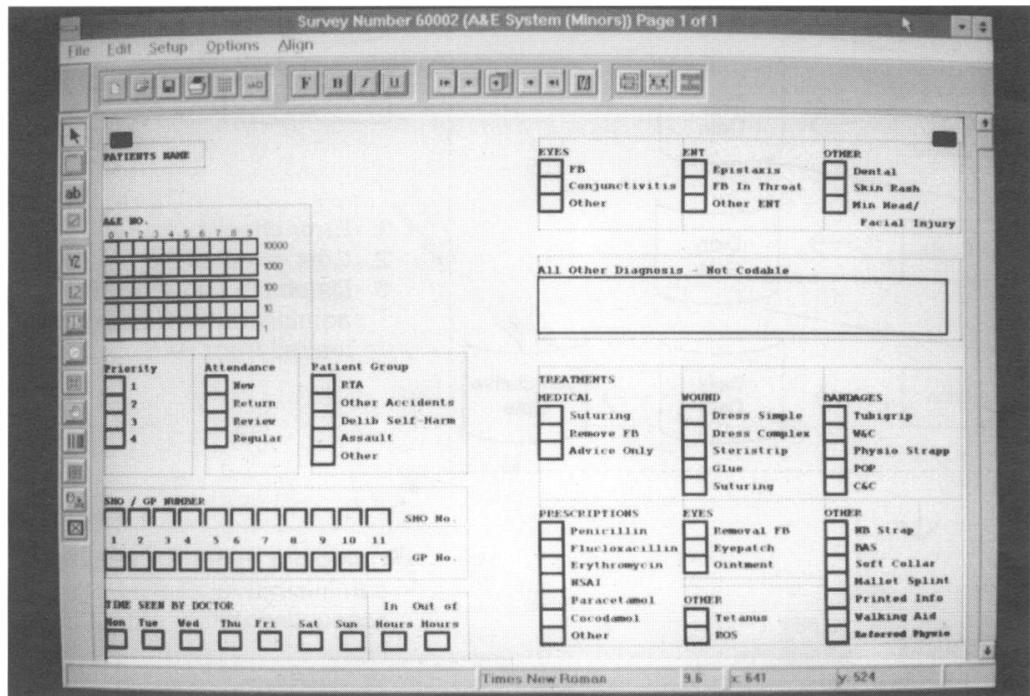

Figure 3 Designing and editing the questionnaires on screen. coded and only $5-15 \%$ need to be written long-hand.

Formic allows the forms to be created by making the screen represent what the page will look like when printed (fig 3). This enables tick boxes and other data collection types (boxes for handwriting, horizontal and handwritten number grids, code frames, bar codes, etc) to be placed anywhere on the page. Choosing appropriate data types and grouping logically linked questions together (that is, creating a single or multiple choice question) has allowed us to build error checks directly into the design of the form. This improves data accuracy by eliminating some of the errors that occur due to faulty coding when the forms are filled in.

Figure 4 shows the finished results. Despite the high detail, all information relating to an attendance can still be collected on a single sheet of A4. The boxed areas on the sheet allow for additional information to be entered long hand. During scanning, these areas are stored as images and appear on screen where they may be manually transferred into typed text. This is then stored in the respective data field. For the present study, the master copies were photocopied, as printing relatively small batches would have been more expensive.

\section{From scanning to database}

The completed forms are collected and may be scanned any time. For our department, which sees between 150 and 200 patients per day, scanning a daily workload takes approximately 20 minutes. At the end of scanning, the operator brings up those stored images onto screen to correct errors and transfer handwritten information into typed text (fig 5). This takes approximately 30 minutes per 100 sheets of data, even when the degree of accuracy which the forms have been filled in is only moderately high, as is usual for busy doctors.

The resulting data files are then sent to Microsoft Access where they go through a series of checks to ensure data accuracy. Duplicates are eliminated, missing consecutive numbers are flagged, and non-consecutive numbers are checked to ensure that they are reattenders or clinic patients. Any patient details that fail on these tests are sent to an errors section to be checked manually.

\section{From database to accounting}

By now, all patient related information required for costing attendance episodes is stored in Access (fig 6); the remainder of the information required is held in the form of reference tables within an Excel spreadsheet.

The bulk of the data are those derived from the workload management study we referred to previously. In brief, this entailed setting standards on management of a given "presenting complaint". The standards took the form of a list of "activities" (take history, examine, take blood, etc) which were further broken down into their individual components. Each activity was timed and the sum of times tabled for medical, nursing, and clerical staff respectively 

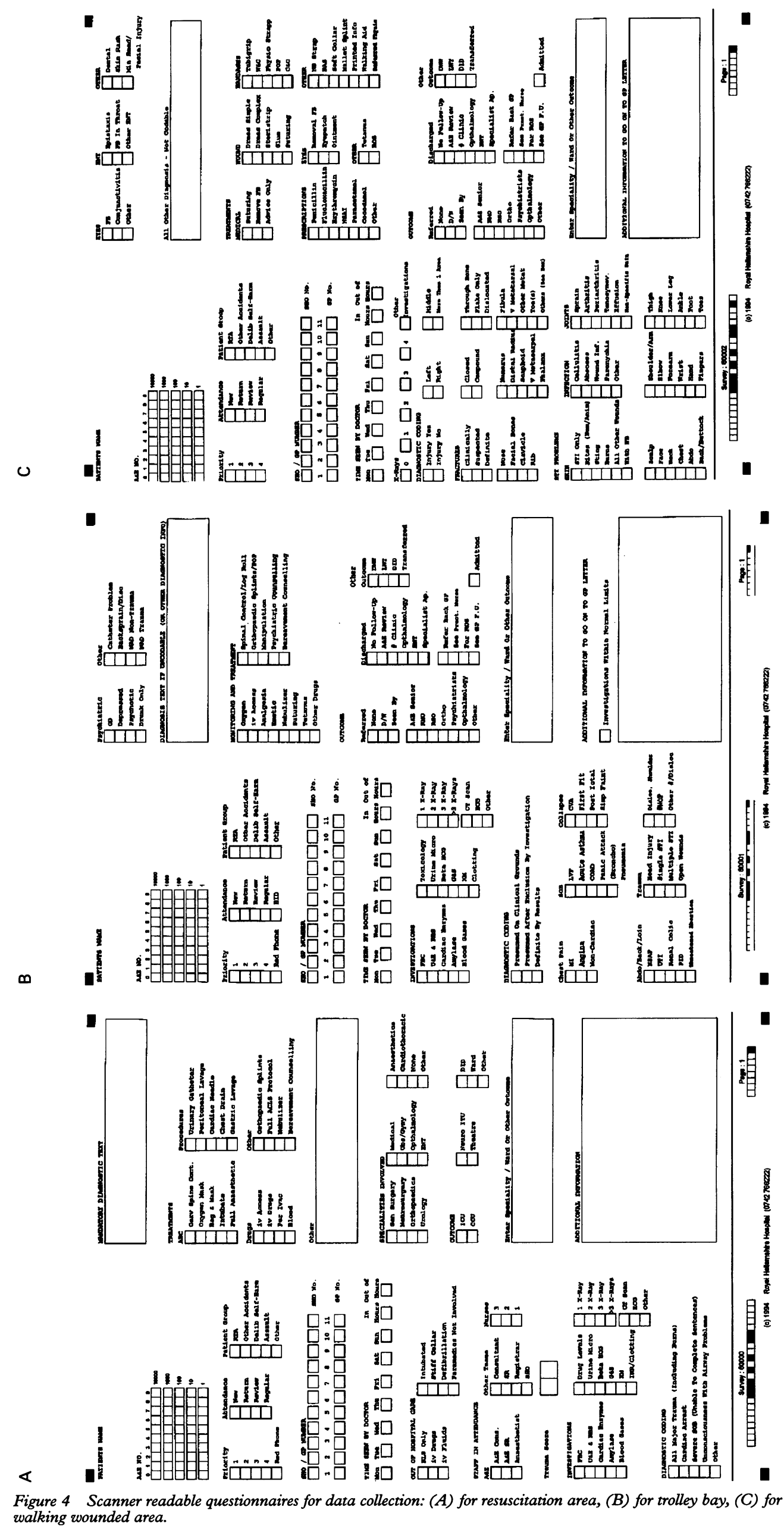


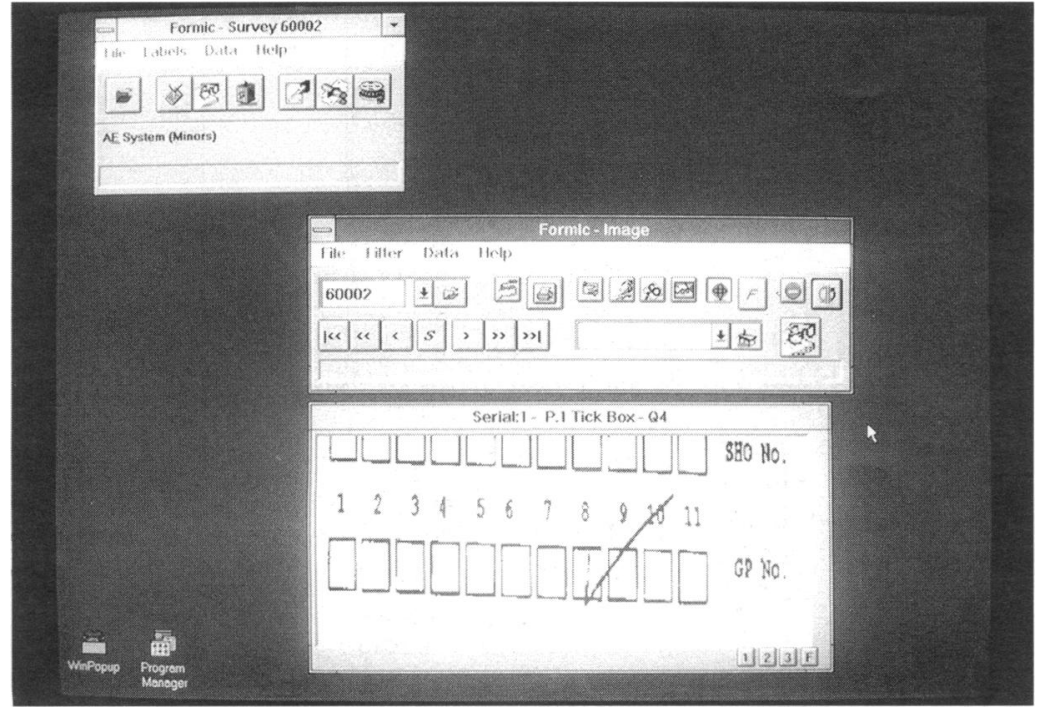

Figure 5 On screen editing of errors and transfer of handwritten information into respective data fields.

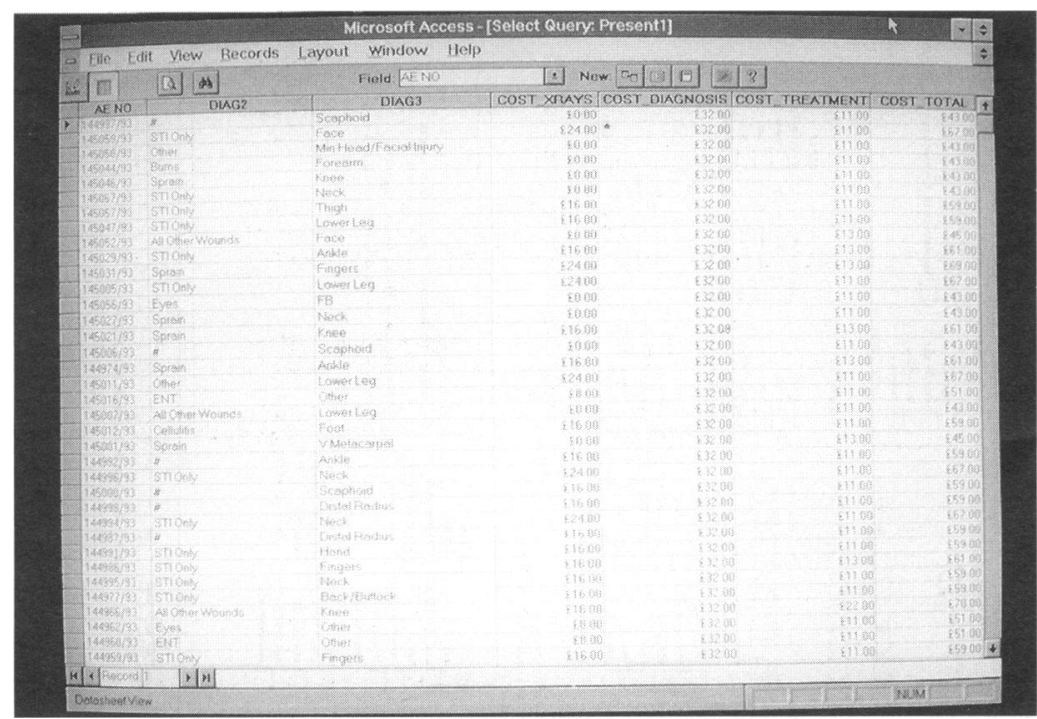

Figure 6 Computer screen showing appearance of Access database record with details of costing of individual patient episodes.

(for further details see previous article). These tables were entered into Excel and linked to tables that hold further information on the cost of wages, $x$ rays, laboratory investigation, sterile supplies, drugs, overheads, and fixed capital costs. Updating one item on any one of the tables automatically updates all related information. Access uses those tables for reference to automatically calculate the cost of treatment of an individual attendance.

At the end of the process, the data are merged with patient identification data downloaded from PAS and then optionally stored on magnetic tape for safekeeping. Each record within the database now contains all administrative (name, postcode, address, GP, etc), clinical (diagnosis, treatments, outcomes), non-clinical (date and time of attendance, triage category, number and specialty of doctors involved, etc) and all cost information (basic, $x$ ray, investigations, etc) for a patient episode.

All regularly required information is extracted by running prewritten macros (computer jargon for smaller, user written programs running within a larger program); nonstandard queries may be designed on screen and then run. Access has extensive facilities for interrogating the database: any data field may be related to any other and the possibilities of extracting information is only limited by the type of data chosen to be kept.

\section{Discussion}

There is little doubt that a system like the one described above is useful: an extensive database containing accurate administrative, clinical, and financial information is an invaluable tool for clinicians and business managers alike. Its remarkable flexibility makes it suitable for the ever changing environment of the new NHS and its ease of use and its speed makes it attractive for use by less computer literate staff who may require information for research, audit, or costing exercises. Although most $A \& E$ departments will still be block contracted, those who can provide their purchasers with accurate coatings of individual treatment episodes will have the edge when negotiating funding. But the system does cost around $£ 16000$ (plus cost of carrying out the workload management studies and recurrent costs for maintenance) and the question is: Is it worth the investment in time and money?

We described the hardware and software we used, all of which were chosen because they were "right" rather than "cheap". The hardware specifications are way above minimum requirement to run the programs. This makes provision for expansion without having to upgrade; we assumed that purchasers will continue to demand increasing amounts of data as proof of quality of service. A tape streamer is the lazy computerist's way of creating backup copies, but its cost is negligible compared to the cost of losing an irreplaceable database. Admittedly, the 17' VGA screen is a luxury, but it does make designing new queries to interrogate the database quicker, easier, and more fun. One of the aims of the project was to create a system that is attractive to those who still view computers with suspicion. We hope that it will provide the incentive for them to realise their ideas for audit and research.

The most expensive items are the scanner and the optical mark reader software, which together account for $75 \%$ of the total cost. Again, despite the high investment, it was the better choice.

The advantages of a scanner over hand entering information are obvious: human error is minimised and it is quicker. The workload management study carried out in our department showed that it takes 27 hours a week of clerical staff time to enter the clinical information into our existing system ${ }^{1}$ and with single operator entry we fall way short of the desirable $99 \%$ mark of data accuracy (this would require double entry of all data by a second clerk with flagging of non-concordant entries). We estimate that once the system is implemented, this will fall to less than 10 hours per week while simultaneously storing more detailed information at the required accuracy. 
With that, the system fulfils two of the most notable criteria for an automated data entry system: above $99 \%$ data accuracy and cost savings after 6-12 months of use. ${ }^{2}$

The second major cost item is the investment in staff time over and above the time spent on data entry: the time required to create and maintain the database. However, enabling departments to design their own data collection forms on-site has distinct advantages. During the design stage, a small team of in-house staff working close to the shop floor will be able to progress using the small steps/frequent corrections approach: regular feedback on intermediate results allows them to arrive relatively quickly at a final design that accurately reflects patient flow and case mix specific to the particular unit. This in turn makes it easy for staff to fill in the completed designs: despite the apparent complexity of our three data collection forms, the acceptance among medical staff was high and consequently the forms were filled with a surprising degree of accuracy and care. Although staff are required to give more information than for our existing PAS module, it does not take longer to gather it.

Furthermore, requirements vary not only between departments, but over time they are likely to vary within the same department. The system allows end users to alter the database themselves using resident expertise; this makes them independent from software companies, thus saving time and money. Theoretically it is now possible to decide upon examining the relation between blue eyes, postcode, and $\mathrm{L}$ ankle injury and start collecting the new data "eye colour blue" a few hours later as part of the routine data collection. Although the practical value of such a project is doubtful, the same could be said for the previously unthinkable, but now nationwide, effort to stopwatch the minutes from arrival to triage.

The idea of the project was derived from the need to develop an accurate and flexible database for accounting, but much of the information required to prepare accounts is clinical, which is now also available for audit and research. Microsoft Access is a general purpose database and data manipulation requires little effort. Its scope is almost unlimited and its remarkable speed encourages asking questions, if for no better reason than curiosity: none of us would have thought that we spend the equivalent of two F grade sister's posts per year to pay for staff to do nothing else but communicate information about patients from one to another-that is not counting the time they spend talking to patients. The reasons are as yet unclear, but we're working on it.

We wish to thank the long suffering staff in Medical Audit for their patience when we insisted that ninety nine point nine percent perfect was just not perfect enough.

1 Taylor CJ, Bull F, Burdis C, Ferguson DG. Workload management in A\&E: counting the uncountable and predicting the unpredictable. J Accid Emerg Med 1997;14:88-91.

2 Emberton M, Rockall T, Meredith P. Scanning for audit. Br J Hosp Comput Inform Manage 1993;10:32-4. 